\title{
Investigating Transformation of CFCs at a Contaminated Field Site Using Compound-Specific Isotope Analysis
}

Elizabeth Phillips1, TeTYANA GILEVSKA1, AXEL HORST1, JESSE MANNA1, EDWARD S. SEGER2, EdWARD J. LUTZ2, SCOTT NORCROSS3, SCOTT A. MORGAN3, KATHRYN A. WEST3, E. ERIN MACK4, SANDRA DWORATZEK5, JENNIFER WEBB5, BARBARA SHERWOOD LOLLAR1*

1Dept of Earth Sciences, Univ. of Toronto, Canada *correspondence: barbara.sherwoodlollar@utoronto.ca 2 Corporate Remediation Group, The Chemours Company, United States 3AECOM, United States

4Corporate Remediation Group, E.I. DuPont de Nemours and Company, United States ${ }_{5}$ SiREM, Canada

Compound-specific isotope analysis (CSIA) is a powerful tool that can be applied to investigate contaminant transport and transformation in field settings. The extent of contaminant transformation can be estimated using the carbon isotopic composition $\left(\delta_{13} \mathrm{C}\right)$ in contaminant molecules and an experimentally-determined enrichment factor $(\varepsilon)$. Controls on contaminant concentrations can be further evaluated in the context of pure-phase $\delta{ }_{13} \mathrm{C}$ signatures to differentiate between $\delta_{13} \mathrm{C}$ that are likely reflective of source or degraded material. Chlorofluorocarbons (CFCs) are contaminants that are amenable to anaerobic biotransformation. However, low environmental concentrations has hindered the application of CSIA to CFCs. This study [1] uses a recently developed technique [2] to determine biotransformation-associated enrichment factors of 1,1,2-trichloro-1,2,2-trifluoroethane (CFC-113) and trichlorofluoromethane (CFC-11) by a mixed enrichment culture. $\delta{ }_{13} \mathrm{C}$ of pure-phase $\mathrm{CFCs}$ were also measured. These results were integrated with CFC concentrations and $\delta_{13} \mathrm{C}$ from a field site to investigate lines of evidence for potential in situ transformation. The results for CFC-11 suggest in situ transformation is likely occurring at the site, with a maximum estimate of transformation up to $86 \%$. In contrast, the results for CFC-113 are inconsistent with in situ transformation. This study provides the first biotransformation-associated $\varepsilon s$ for CFCs and significantly expands the database of known pure-phase $\delta_{13} \mathrm{C}$ to compare to field measurements.

[1] Phillips et al. (2020), Environ. Sci. Technol. 54, 870-878.

[2] Horst et al. (2015), Anal. Chem. 87, 10498-10504. 